ARTICLE

\title{
Angiotensin inhibition enhances drug delivery and potentiates chemotherapy by decompressing tumour blood vessels
}

Vikash P. Chauhan ${ }^{1,2, \star}$, John D. Martin ${ }^{1,3, *}$, Hao Liu ${ }^{1,4}$, Delphine A. Lacorre ${ }^{1}$, Saloni R. Jain ${ }^{1,3}$, Sergey V. Kozin ${ }^{1}$, Triantafyllos Stylianopoulos ${ }^{1,5}$, Ahmed S. Mousa ${ }^{1}$, Xiaoxing Han ${ }^{1}$, Pichet Adstamongkonkul,2, Zoran Popović 6 , Peigen Huang ${ }^{1}$, Moungi G. Bawendi ${ }^{6}$, Yves Boucher ${ }^{1} \&$ Rakesh K. Jain ${ }^{1}$

Cancer and stromal cells actively exert physical forces (solid stress) to compress tumour blood vessels, thus reducing vascular perfusion. Tumour interstitial matrix also contributes to solid stress, with hyaluronan implicated as the primary matrix molecule responsible for vessel compression because of its swelling behaviour. Here we show, unexpectedly, that hyaluronan compresses vessels only in collagen-rich tumours, suggesting that collagen and hyaluronan together are critical targets for decompressing tumour vessels. We demonstrate that the angiotensin inhibitor losartan reduces stromal collagen and hyaluronan production, associated with decreased expression of profibrotic signals TGF- $\beta 1$, CCN2 and ET-1, downstream of angiotensin-II-receptor-1 inhibition. Consequently, losartan reduces solid stress in tumours resulting in increased vascular perfusion. Through this physical mechanism, losartan improves drug and oxygen delivery to tumours, thereby potentiating chemotherapy and reducing hypoxia in breast and pancreatic cancer models. Thus, angiotensin inhibitors inexpensive drugs with decades of safe use - could be rapidly repurposed as cancer therapeutics.

\footnotetext{
${ }^{1}$ Edwin L. Steele Laboratory, Department of Radiation Oncology, Massachusetts General Hospital and Harvard Medical School, Boston, Massachusetts 02114, USA. ${ }^{2}$ School of Engineering and Applied Sciences, Harvard University, Cambridge, Massachusetts 02138, USA. ${ }^{3}$ Department of Chemical Engineering, Massachusetts Institute of Technology, Cambridge, Massachusetts 02139, USA. ${ }^{4}$ Program in Biological and Biomedical Sciences, Harvard Medical School, Boston, Massachusetts 02115, USA. ${ }^{5}$ Department of Mechanical and Manufacturing Engineering, University of Cyprus CY-1678, Nicosia, Cyprus.

${ }^{6}$ Department of Chemistry, Massachusetts Institute of Technology, Cambridge, Massachusetts 02139, USA. * These authors contributed equally to this work. Correspondence and requests for materials should be addressed to R.K.J. (email: jain@steele.mgh.harvard.edu).
} 
D rug and oxygen delivery in tumours is dependent on the organization and efficiency of perfused vessels ${ }^{1-3}$. Solid stress (pressure from solid tissue components) accumulates in tumours as cancer and stromal cells proliferate in a confined microenvironment created by crosslinked matrix molecules ${ }^{4-6}$. The matrix stores and transmits this stress throughout the tumour ${ }^{7}$, collapsing blood vessels to limit perfusion $^{8,9}$. Meanwhile, patients with low tumour perfusionpresumably with extensive hypoxia and impaired drug deliveryshow poorer chemotherapy responses and shorter survival versus patients with high perfusion ${ }^{10,11}$. Thus, there is an urgent need for drugs that can target solid stress to improve blood perfusion and drug delivery in tumours.

Stromal cells and matrix contribute to solid stress, but the mechanisms through which these components interact to compress tumour vessels remain unclear. Uncovering these mechanisms could lead to novel paradigms for enhancing tumour perfusion. In this study, we show that different tumour matrix components produced by cancer-associated fibroblasts (CAFs) collaborate to compress vessels. We demonstrate that angiotensin signalling blockade inactivates CAFs to reduce their production of these matrix components while also reducing CAF density. Through this mechanism, we find that angiotensin receptor blockers (ARBs) reduce solid stress, decompress tumour vessels, increase drug and oxygen delivery and improve chemotherapy outcomes. Thus, solid stress can be targeted by decreasing CAF activity and agents that do so can improve tumour perfusion and enhance chemotherapy.

\section{Results}

Hyaluronan and collagen collaborate to compress tumour blood vessels. Hyaluronan has been proposed to be the primary matrix molecule responsible for vessel compression ${ }^{12}$, but its exact mechanism remains unclear. Hyaluronan interacts with collagen in a complex manner ${ }^{13}$, and both contribute to the accumulation of solid stress through these interactions ${ }^{5}$. Thus, we examined whether collagen may also affect vessel compression (Fig. 1a,b). We measured vessel perfusion (patency) in multiple orthotopic tumours and compared it with hyaluronan and collagen levels. Interestingly, we found that both hyaluronan and collagen area fraction were inversely associated with perfused vessel fractions (Fig. 1c). The mean of these matrix area fractions had an even stronger inverse correlation with perfusion, indicating a complex nonlinear relationship between the two matrix molecules and perfusion. Surprisingly, we found that vessel perfusion did not correlate with hyaluronan area fractions in tumours with low collagen levels (Fig. 1d); rather, hyaluronan and perfusion were inversely correlated only in collagen-rich tumours. In contrast, perfusion inversely correlated with collagen area fraction in both hyaluronan-rich and hyaluronan-poor tumours (Fig. 1e). These findings indicate that collagen is needed for hyaluronan to exert compression on blood vessels, suggesting that both are potential targets for vessel decompression in desmoplastic tumours.

Angiotensin inhibition reduces stromal fibrosis signalling. We therefore sought a translatable therapy that targets both collagen and hyaluronan. We recently found that the ARB losartan can reduce collagen I production in CAFs by downstream inhibition of transforming growth factor (TGF)- $\beta 1$ activation ${ }^{14,15}$ through thrombospondin-1 (TSP-1) inhibition ${ }^{16-18}$. As hyaluronan synthesis is similarly TGF- $\beta 1$ dependent, we expected that losartan would also decrease hyaluronan production. We generated transgenic FVB mice expressing the fluorescent protein dsRed driven by the promoter for $\alpha$-smooth-muscle actin $(\alpha \mathrm{SMA})^{19}$, a marker for activated CAFs. In these mice $\left(\alpha S M A^{P}\right.$-dsRed/FVB), we orthotopically implanted AK4.4 pancreatic tumour cells, treated these mice with losartan or saline (control), and then isolated the CAFs (activated pancreatic stellate cells) from their tumours using fluorescence-activated cell sorting (Supplementary Fig. S1). We confirmed that CAF expression of TGF- $\beta 1$ and collagen I was reduced by losartan (Fig. 2a,b). We then measured expression of hyaluronan synthases $1-3$ in these CAFs and found that hyaluronan synthases 1-3 were also greatly decreased by losartan (Fig. 2a,b). Thus, losartan is a dual inhibitor of stromal collagen and hyaluronan production.

Losartan rapidly reduces established tumour matrix levels ${ }^{14}$, which implies that it degrades or destabilizes matrix through an uncharacterized mechanism in addition to preventing matrix production through TGF- $\beta 1$. Connective tissue growth factor (CCN2/CTGF), a matricellular protein, stabilizes the transient fibrosis produced by TGF- $\beta 1$ activity ${ }^{20,21}$ and is downstream of angiotensin signalling through TGF- $\beta 1$ in other fibrosis diseases $^{22,23}$, suggesting that losartan could be destabilizing the matrix by inhibiting CCN2 expression. By comparing CAFs isolated from losartan- versus saline-treated mice, we found that losartan reduced expression of CCN2 (Fig. 2a,b). We next used immunohistochemistry to assess how losartan affects CCN2, active TGF- $\beta 1$ and matrix levels in orthotopic E0771 and AK4.4 models, which recapitulate the desmoplasia observed in human mammary adenocarcinoma and in human pancreatic ductal adenocarcinoma, respectively (Supplementary Figs S2-S4). Losartan reduced both CCN2 and active TGF- $\beta 1$ in E0771 breast and AK4.4 pancreatic tumours (Fig. 2c-f and Supplementary Fig. S5). Further, losartan decreased collagen I and hyaluronan in both of these tumours (Fig. 3a-d and Supplementary Figs S4, S6 and S7). Thus, losartan decreases stromal matrix production through TGF- $\beta 1$ while potentially destabilizing the existing matrix through CCN2. Collectively, these effects lead to a reduction in collagen and hyaluronan levels in desmoplastic tumours.

Angiotensin inhibition decreases CAF density in tumours. As the effects of losartan on matrix expression in tumours were pronounced, and as angiotensin signalling has been previously shown to activate fibroblasts in other fibrosis diseases 24,25 , we next tested whether losartan could also reduce CAF density. We found that losartan lowered the density of $\alpha$ SMA + cells in E0771 and AK4.4 tumours (Fig. 3e,f and Supplementary Fig. S8), indicating a decrease in activated CAFs. In other fibrotic diseases, fibroblast activation occurs through endothelin-1 (ET-1) expres$\operatorname{sion}^{26}$ downstream of TGF- $\beta 1^{27}$ and angiotensin signalling 28 Thus, we expected that losartan may reduce ET-1 expression by CAFs. Indeed, in the CAFs isolated from losartan- or salinetreated mice, we found that losartan decreased the expression of ET-1 (Fig. 2a,b). Therefore, losartan seems to reduce activation of fibroblasts or stellate cells to $\alpha \mathrm{SMA}+$ CAF phenotype, leading to a reduction in CAF density.

AT1 and AT2 signalling opposingly affect tumour fibrosis. To investigate how best to target angiotensin signalling, we next investigated the role of angiotensin receptor signalling in tumour desmoplasia. We first studied the effects of the angiotensin-converting enzyme inhibitor (ACE-I) lisinopril on the matrix, because ACE-Is block both angiotensin-II-receptor-1 (AT1) and angiotensin-II-receptor-2 (AT2) signalling. Intriguingly, lisinopril reduced collagen I and hyaluronan in AK4.4 tumours to a lesser extent than losartan, which blocks only AT1 signalling (Supplementary Fig. S9). This suggests that AT2 inhibition leads to increased matrix levels in tumours, in opposition to AT1 inhibition. We confirmed this by implanting E0771 in knockout 


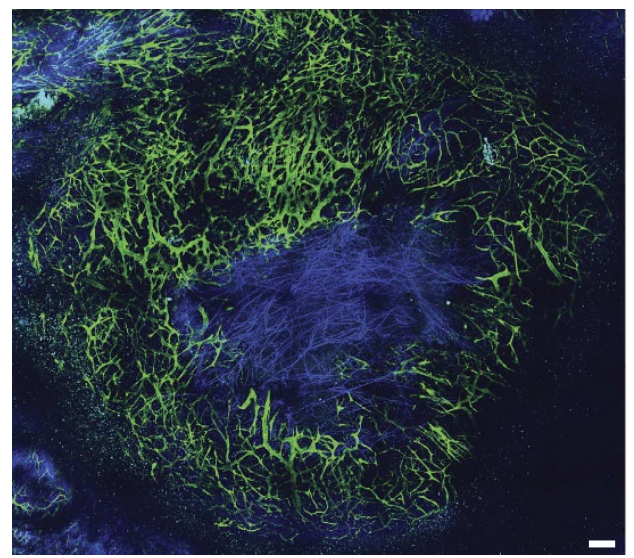

Collagen perfused vessels
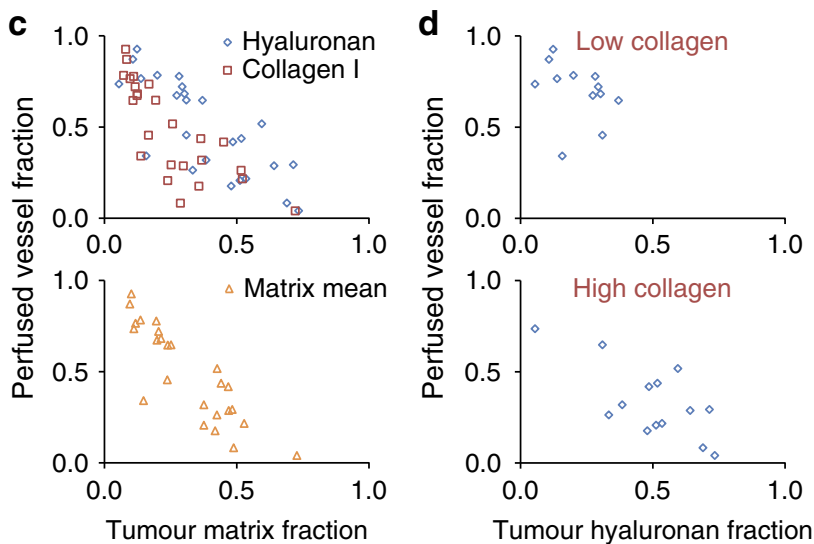
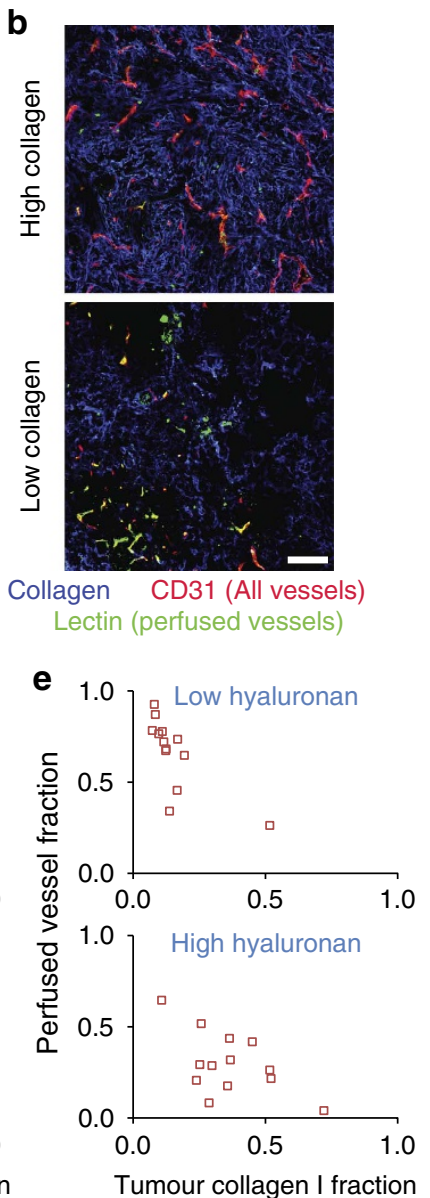

Figure 1 | Collagen and hyaluronan interact to compress tumour blood vessels. (a) Representative image from intravital multiphoton microscopy of perfused tumour vessels (green) and collagen (blue), showing that high collagen levels colocalize with low perfusion in an E0771 breast tumour. Scale bar, $200 \mu \mathrm{m}$. (b) Histology images of vascular perfusion in orthotopic AK4.4 pancreatic tumours with high versus low collagen levels. High local collagen I levels (blue) appear to colocalize with collapsed vessels (red, collapsed; green/yellow, perfused) in vivo. Scale bar, $100 \mu \mathrm{m}$. (c) Correlation of perfused vessel fraction versus tumour matrix area fractions in multiple orthotopic pancreatic tumour models (AK4.4 and L3.6pl) in mice. Following lectin injection and animal killing, perfusion was quantified as the fraction of vessels that are both lectin- and CD31-positive out of all CD31-positive vessels. Perfusion inversely correlates with both hyaluronan $(R=-0.79, P<0.001$, Pearson's correlation) and collagen I $(R=-0.78, P<0.001$, Pearson's correlation), but has a stronger inverse correlation $(R=-0.86, P<0.001$, Pearson's correlation) with the average matrix area fraction. (d) Grouping these tumours into those with either low $(<17 \%)$ or high $(\geq 17 \%)$ collagen reveals that perfusion does not correlate $(R=-0.33$, Pearson's correlation) with hyaluronan in collagen-poor tumours but does inversely correlate $(R=-0.71, P=0.004$, Pearson's correlation) in collagen-rich tumours. (e) In contrast, grouping the tumours into those with either low $(<33 \%)$ or high $(\geq 33 \%)$ hyaluronan shows that perfusion inversely correlates with collagen I in both hyaluronan-poor $(R=-0.73, P=0.003$, Pearson's correlation) and hyaluronan-rich $(R=-0.57, P=0.040$, Pearson's correlation) tumours.

mouse models of AT1 (Agtr1a $\left.a^{-/-}\right)$and AT2 (Agtr2 $\left.{ }^{-/-}\right)$. We found that tumours in the AT1-knockout mice had lower levels of collagen I and hyaluronan than tumours in wild-type mice (Fig. 4a,b), whereas the tumours in AT2-knockout mice had higher collagen I and hyaluronan levels than tumours in wildtype mice (Fig. 4c,d), indicating that stromal AT2 signalling inhibits tumour desmoplasia, whereas AT1 signalling promotes it. Importantly, this result fits with the known effects of angiotensin signalling on fibrosis in other disease ${ }^{29-31}$. To confirm the relevance of stromal AT1 and AT2 inhibition, we measured the expression of these receptors in CAFs and cancer cells from wildtype mice. We found that CAFs isolated from AK4.4 tumours express far more AT1 and AT2 than AK4.4 or E0771 cancer cells (Fig. 4e). We confirmed this result with immunohistochemistry in E0771 tumours, where we found that a moderate fraction of CAFs in these tumours express AT1 at high levels, whereas most other cells express AT1 at low levels; most CAFs express high levels of AT2, whereas a small fraction of other cells express similar AT2 levels (Fig. 4f,g). These results indicated that the cellular targets of angiotensin blockers relating to matrix production are CAFs rather than cancer cells. Thus, the antidesmoplasia benefits of ARBs are because of specific AT1 inhibition in tumour stroma, and ACE-Is may not provide the same benefits due to combined AT1 and AT2 inhibition.

Angiotensin inhibition lowers solid stress in tumours. We next investigated the effects of losartan on solid stress in tumours. We measured solid stress in these tumours using our recently established technique ${ }^{5}$. Losartan reduced solid stress in both E0771 and AK4.4 tumours (Fig. 5a), as well as in 4T1 breast and Pan-02 pancreatic tumours (Fig. 5b). We additionally tested a panel of ARBs and found that they all reduced solid stress (Supplementary Fig. S10), further suggesting that the mechanism of action is via AT1 inhibition rather than an off-target effect of losartan. These data indicate that angiotensin blockers may be capable of 
a

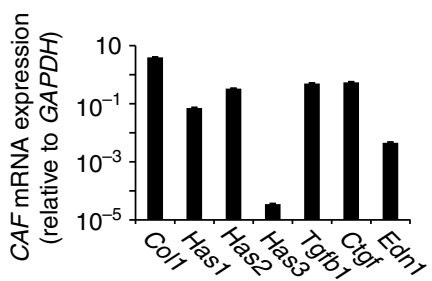

b

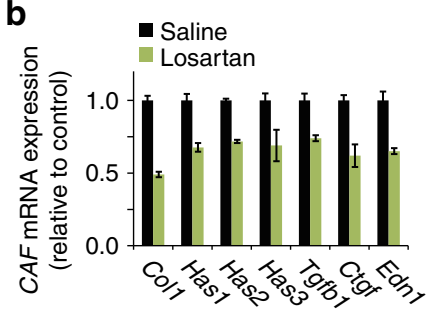

C

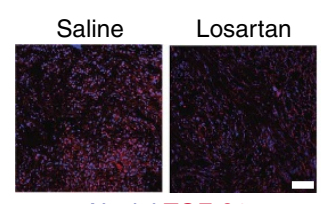

Nuclei TGF- $\beta 1$

d

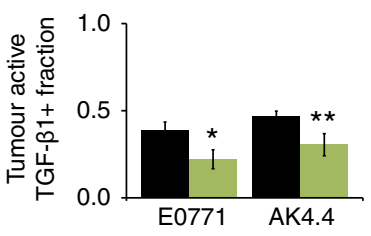

e

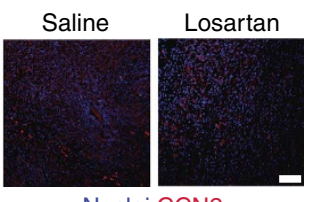

Nuclei CCN2

Figure 2 | Losartan decreases profibrotic stromal activity in tumours. (a) Expression levels of fibrosis-related genes in murine cancer-associated fibroblasts (CAFs) isolated from orthotopic AK4.4 pancreatic tumours. AK4.4 tumours were orthotopically implanted in $\alpha S M A^{P}$-dsRed/FVB mice, resulting in $\alpha \mathrm{SMA}+\mathrm{CAFs}$ that express dsRed. These mice were treated with losartan or saline (control), then the CAFs from their tumours were isolated using fluorescence-activated cell sorting for dsRed. The isolated control CAFs express high mRNA levels of collagen I (Col1), hyaluronan synthases (HAS)1-2 (Has1, Has2), TGF- $\beta 1$ (Tgfb1) and CCN2 (Ctgf), with low levels of HAS 3 (Has3) and moderate levels of ET-1 (Edn1). Levels normalized to GAPDH expression. (b) Comparison of expression levels of fibrosis-related genes in the CAFs isolated from losartan- or saline-treated (control) mice. Losartan reduces the mRNA expression of each of these profibrotic genes in CAFs $(P<0.05$, Student's $t$-test). Levels normalized to saline control. (c) Histology images showing the effect of losartan on tumour TGF- $\beta 1$ expression. Scale bar, $100 \mu \mathrm{m}$. (d) Immunohistochemical analysis of TGF- $\beta 1$ expression with losartan treatment. Losartan reduces the expression of TGF- $\beta 1$ in E0771 ( ${ }^{\star} P=0.048$, Student's $t$-test) and AK4.4 tumours ( ${ }^{\star \star} P=0.044$, Student's $t$-test). (e) Histology images showing the effect of losartan on tumour CCN2 expression. Scale bar, $100 \mu \mathrm{m}$. (f) Immunohistochemical analysis of CCN2 expression with losartan treatment. Losartan reduces the expression of CCN2 in E0771 ( ${ }^{\star} P=0.044$, Student's $t$-test) and AK4.4 ( ${ }^{\star \star} P=0.046$, Student's $t$-test) tumours. Scale bar, $100 \mu \mathrm{m}$. Animal numbers $n=4$ (CAF expression), $n=7-8$ (E0771 TGF- $\beta 1, C C N 2), n=4-6$ (AK4.4 TGF- $\beta 1$, CCN2). Error bars indicate s.e.m.

decompressing vessels in desmoplastic tumours by reducing solid stress through their antimatrix effects.

Angiotensin inhibition decompresses tumour blood vessels. We hypothesized that the reduction in solid stress by losartan would increase vascular perfusion in our desmoplastic tumour models. We found these tumours to be severely hypoperfused (Supplementary Fig. S4): only 23\% of vessels in E0771 breast tumours and $21 \%$ in AK4.4 pancreatic tumours were perfused with blood (Fig. 6a,b). Of note, collagen-rich areas seemed to colocalize with low-perfusion areas (Fig. 3a). Losartan significantly improved the perfused vessel fraction to $43 \%$ in E0771 and $45 \%$ in AK4.4 (Fig. 6a,b). Losartan treatment also increased the fraction of vessels with open lumen in E0771 and AK4.4 (Fig. 6c), further indicating vascular decompression as the mechanism of action ${ }^{9}$. Interestingly, the open lumen fraction was higher than the perfused vessel fraction in both models-perhaps because of collapse or occlusion in feeding vessels or flow cessation and clot formation ${ }^{1}$. Importantly, losartan did not significantly improve perfusion in the well-perfused L3.6pl tumour model, which has low collagen and $68 \%$ vessel perfusion at baseline (Supplementary Fig. S11), confirming the findings of our previous study ${ }^{14}$. Thus, losartan can increase blood perfusion in tumours by opening existing collapsed blood vessels.

Despite the previous classification of angiotensin inhibitors as antiangiogenics ${ }^{32}$, we found that losartan did not reduce vascular endothelial growth factor (VEGF) levels (Supplementary Fig. S12), affect the CD31 + vessel density in these tumours (Fig. 6d,e) or decrease vessel diameter (Supplementary Fig. S13). Further, losartan increased the effective permeability of the blood vessels for all sizes of nanoparticles (Supplementary Fig.
S14), which is inconsistent with the 'vascular normalization' effect that results from antiangiogenic therapy ${ }^{33}$. Moreover, this dose did not decrease blood pressure in a separate group of tumourbearing mice with advanced disease (Supplementary Fig. S15) ${ }^{14}$, and correspondingly did not affect flow rates in individual blood vessels (Supplementary Fig. S16). As such, we confirmed that losartan does not improve perfusion by reducing vessel leakiness or increasing blood flow, as seen after vascular normalization ${ }^{11,33}$, but rather does so through 'microenvironmental normalization'.

Angiotensin inhibitors enhance drug and oxygen delivery. As drug and oxygen delivery to tissues is governed by vascular perfusion, we tested the effects of losartan on delivery to tumours. We first engaged a mathematical approach to analyse the efficiency of the vascular network for drug and oxygen delivery. Using multiphoton microscopy, we imaged the perfused vessel networks of E0771 tumours (Fig. 6a). On the basis of metrics of intervascular spaces $^{34}$, we found that losartan reduces the distance drugs and oxygen must travel to reach tumour cells (Fig. $6 \mathrm{f}$ and Supplementary Fig. S17). We then measured the total tissue accumulation of the small-molecule chemotherapeutic fluorouracil (5-fluorouracil; 5-FU). Losartan improved 5-FU delivery to AK4.4 tumours while not affecting delivery to normal organs (Fig. 6g). These data imply that this strategy for enhancing delivery selectively affects tumours, which is expected, since solid stress does not accumulate in most normal organs ${ }^{5}$. We then studied oxygenation using phosphorescence quenching microscopy ${ }^{35}$. We found that losartan treatment maintained tumour oxygen levels in E0771 tumours, whereas control-treated tumours showed a typical growth-dependent drop in oxygenation (Fig. 6h,i). We confirmed this decrease in tumour hypoxia by using pimonidazole staining in E0771 and 4T1 (Fig. 6j and 
a

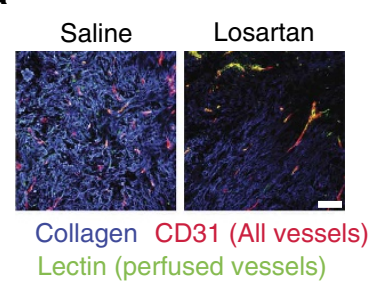

C

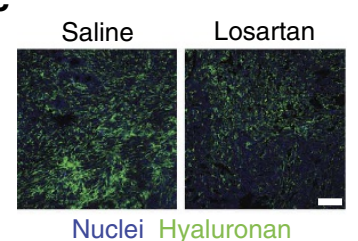

e

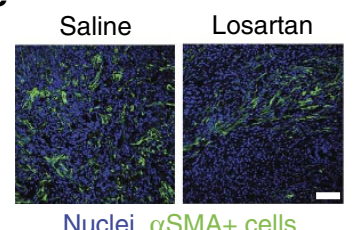

Nuclei $\alpha \mathrm{SMA}+$ cells

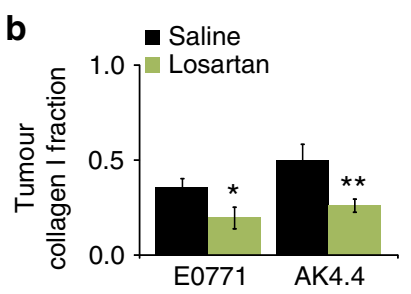

d

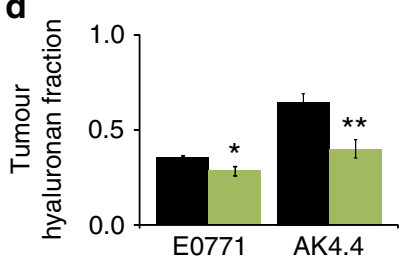

$\mathbf{f}$

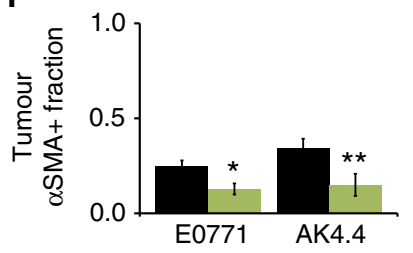

Figure 3 | Losartan reduces matrix and stromal density in tumours. (a) Histology images showing the effect of losartan on tumour collagen levels and perfusion. Scale bar, $100 \mu \mathrm{m}$. (b) Immunohistochemical analysis of tumour collagen levels following losartan treatment. Losartan decreases the collagen I-positive area fraction in E0771 ( ${ }^{\star} P=0.040$, Student's $t$-test $)$ and AK4.4 ( ${ }^{\star \star} P=0.022$, Student's $t$-test) tumours. (c) Histology images showing the effect of losartan on tumour hyaluronan levels. Scale bar, $100 \mu \mathrm{m}$. (d) Immunohistochemical analysis of tumour hyaluronan levels following losartan treatment. Losartan also reduces the hyaluronan-positive area fraction in E0771 ( ${ }^{\star} P=0.048$, Student's $t$-test) and AK4.4 ${ }^{\star \star} P=0.019$, Student's $t$-test) tumours, as assessed using a hyaluronanbinding protein probe. (e) Histology images showing the effect of losartan on tumour aSMA + CAF levels. Scale bar, $100 \mu \mathrm{m}$.

(f) Immunohistochemical analysis of $\alpha \mathrm{SMA}+\mathrm{CAF}$ density with losartan treatment. Losartan reduces the CAF density in E0771 ( ${ }^{\star} P=0.019$, Student's $t$-test) and AK4.4 ( ${ }^{\star \star} P=0.040$, Student's $t$-test) tumours. Animal numbers $n=5-7$ (E0771 collagen), $n=4-6$ (AK4.4 collagen), $n=4-5$ (E0771 hyaluronan), $n=4$ (AK4.4 hyaluronan), $n=5$ (E0771 $\alpha S M A), n=5-6$ (AK4.4 $\alpha$ SMA). Error bars indicate s.e.m.

Supplementary Figs S18 and S19). Thus, reducing solid stress through 'microenvironmental normalization' with angiotensin inhibitors increases the delivery of drugs and oxygen to tumours.

Angiotensin inhibition potentiates chemotherapy. Given these effects on delivery, we tested losartan in combination with doxorubicin in E0771 and 4T1 tumours, or with 5-FU in AK4.4 tumours. We found that the combination of losartan with chemotherapy significantly delayed E0771, 4T1 and AK4.4 tumour growth, whereas monotherapy had no effect (Fig. $7 \mathrm{a}-\mathrm{c}$ and Supplementary Fig. S20). Moreover, the combination of losartan and chemotherapy increased median survival (time to death or excessive tumour burden) for mice bearing E0771 and 4T1 tumours compared with monotherapy (Fig. 7d,e). Similarly, the combination increased median survival (time to death) for mice bearing AK4.4 tumours, whereas monotherapy did not extend survival (Fig. 7f). Importantly, losartan alone-despite increasing blood perfusion-did not shorten survival in any model. The

results of the combination for survival were somewhat better than for primary tumour growth delay, indicating a possible effect on metastases. Correspondingly, losartan slightly decreased metastasis in E0771 (Supplementary Fig. S21), consistent with previous studies $^{36}$ and probably because of the role of angiotensin signalling in promoting the metastatic cascade ${ }^{37,38}$. Of note, we found that losartan combined with doxorubicin delayed tumour growth in both immunodeficient and immunocompetent models of breast cancer (Fig. 7a,b and Supplementary Fig. S21), although the effects appeared less pronounced in immunocompetent mice even at high doses of doxorubicin. This might be attributed to resistance induced by the immune microenvironment ${ }^{39}$. Together, these data demonstrate that angiotensin inhibitors can improve the effectiveness of small-molecule chemotherapeutics through antimatrix effects.

\section{Discussion}

This study highlights the complex role of the tumour matrix as a drug delivery barrier. Although the distribution of larger drugs such as nanomedicines is hindered by steric interactions with collagen, the matrix does not directly limit the distribution of smaller, conventional chemotherapeutics ${ }^{40}$. Rather, the matrix indirectly limits the delivery of conventional chemotherapy through vascular compression. Both hyaluronan and collagen fibres contribute to solid stress in tumours: hyaluronan does so by resisting compression, whereas collagen does so by resisting tension and confining the local microenvironment ${ }^{5}$. Our results lead to a new model for how hyaluronan and collagen affect vessel compression. As cancer and stromal cells proliferate, they attempt to expand their local tumour microenvironment, exerting tensile stress. This tensile stress stretches collagen fibres in the microenvironment, which store tensile elastic 'strain' energy and stiffen, thereby resisting this expansion. The effect is to confine these cells such that the force they generate while proliferating becomes a compressive stress. These cells cannot transmit compressive stress perpendicularly to the fibres of this stiffened collagen; hence, this compressive stress is instead exerted largely on hyaluronan in the local tumour microenvironment. Hyaluronan, whose internal charges repel each other electrostatically and trap water molecules, resists this compression, only storing compressive 'strain' energy until maximally compressed. Beyond this maximal compression, the excess compressive stress is transmitted by hyaluronan to tumour vessels. These tumour vessels, which lack the complete coverage by pericytes and basement membrane that fortifies mature vessels, are structurally weak and cannot resist compression. If collagen levels are low in a tumour, the microenvironment is more easily expanded by proliferating cells and thus compressive stress is not produced to as high a level. As a result, hyaluronan does not contribute to vessel compression in collagen-poor tumours. If hyaluronan levels are low, the compressive stress is not transmitted to vessels to as great a degree. Thus, hyaluronan seems responsible for transmitting compressive stress to vessels in all cases when there is no direct cell contact, whereas collagen enables compressive stress to be applied to hyaluronan by cells.

Analyses of retrospective clinical data suggest that the use of AT1 blockers (ARBs) and ACE-Is to manage hypertension in cancer patients receiving standard therapies is correlated with longer survival in pancreatic and other cancers ${ }^{41-43}$, as well as a reduced risk of recurrence in breast cancer ${ }^{44}$. However, a causal relationship between the use of ARBs/ACE-Is and its clinical benefit-as well as the mechanism behind this potential effect-has not been revealed. AT1 signalling has been shown to increase VEGF expression by CAFs ${ }^{45}$, and both ACE-Is and ARBs can decrease VEGF expression and angiogenesis ${ }^{46,47}$. It has therefore 

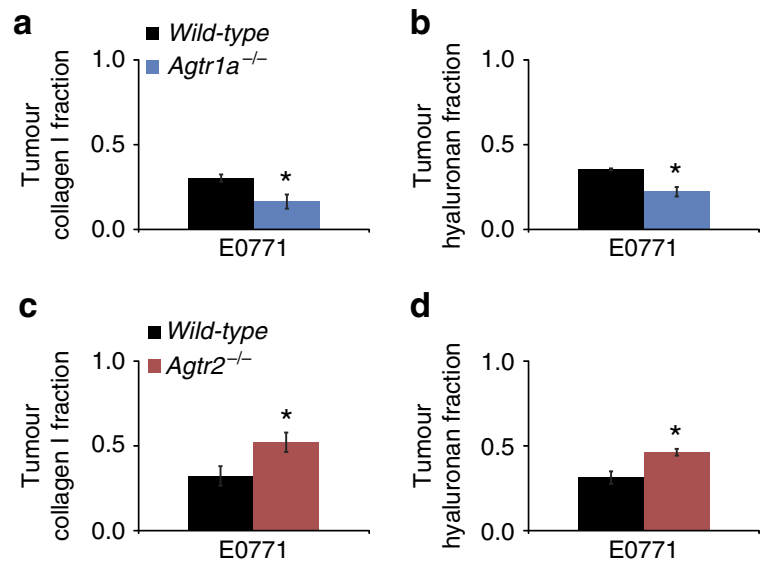

d

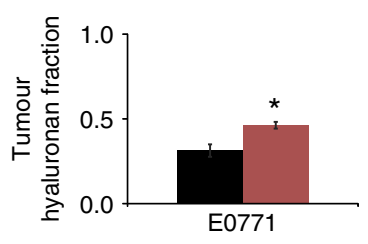

e

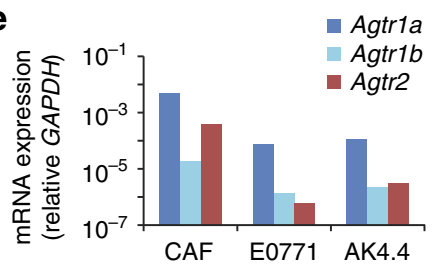

$\mathbf{f}$

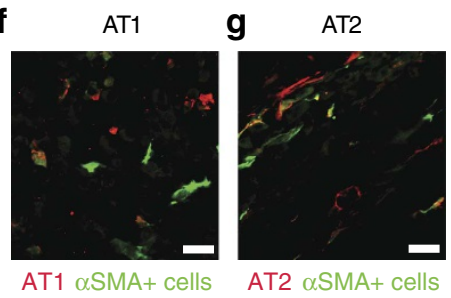

Figure 4 | Stromal angiotensin signalling induces matrix production in tumours. (a) Tumour collagen and (b) hyaluronan levels in angiotensin-IIreceptor-1 (AT1)-knockout mice. Orthotopic E0771 breast tumours implanted in AT1-knockout mice (Agtr1a ${ }^{-}{ }^{-}$) have (a) a lower collagen I area fraction $\left({ }^{\star} P=0.026\right.$, Student's $t$-test) and $(\mathbf{b})$ a lower hyaluronan area fraction ( ${ }^{\star} P=0.011$, Student's $t$-test) than E0771 tumours implanted in wild-type $C 57 \mathrm{BL} / 6$ mice. (c) Tumour collagen and (d) hyaluronan levels in angiotensin-II-receptor-2 (AT2) knockout mice. Orthotopic E0771 breast tumours implanted in AT2knockout mice $\left(\right.$ Agtr $2^{-/}-$) have $(\mathbf{c})$ a higher collagen I area fraction $\left({ }^{\star} P=0.047\right.$, Student's $t$-test $)$ and $(\mathbf{d})$ a higher hyaluronan area fraction $\left({ }^{\star} P=0.012\right.$, Student's t-test) than E0771 tumours implanted in wild-type C57BL/6 mice. Tumours were time- and size-matched at $\sim 200 \mathrm{~mm}^{3}$. (e) Expression of AT1 (Agtr1a and Agtr1b genes) and AT2 (Agtr2 gene) mRNA in murine cancer-associated fibroblasts (CAFs) and cancer cells. CAFs isolated from AK4.4 tumours express over one order of magnitude more AT1 than either E0771 or AK4.4 cancer cells. Similarly, the CAFs express over two orders of magnitude more AT2 than either E0771 or AK4.4 cells. (f) Expression of AT1 (red) in aSMA + CAFs (green) and cancer cells in tumours. We imaged CAFs based on aSMA + expression in E0771 tumours. We found that some CAFs in these tumours express AT1 at high levels, whereas other cells express AT1 at low levels. Colocalization is shown in yellow. Scale bar, $25 \mu \mathrm{m}$. (g) Expression of AT2 (red) in $\alpha$ SMA + CAFs (green) and cancer cells in tumours. Most CAFs express high levels of AT2, whereas some other cells express similar AT2 levels. Colocalization is shown in yellow. Scale bar, $25 \mu \mathrm{m}$. Animal numbers $n=4-5$. Error bars indicate s.e.m.
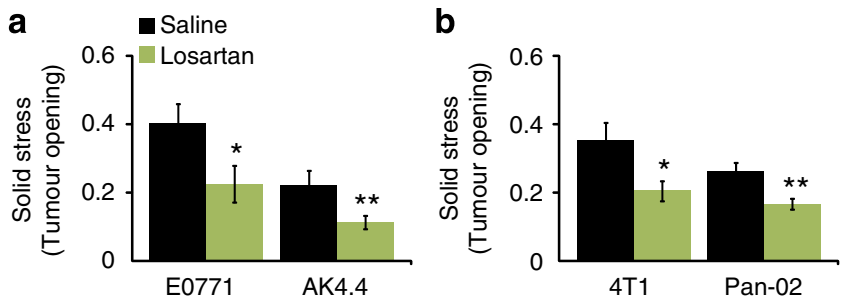

Figure 5 | Losartan targets solid stress in tumours. (a) Solid stress levels in tumours after angiotensin inhibition using losartan. Solid stress was assessed using an ex vivo technique involving the measurement of the extent of tumour tissue relaxation (tumour opening relative to tumour diameter) following a stress-releasing incision, with larger openings indicating higher stress ${ }^{5}$. Through its antimatrix effects, losartan reduces solid stress in E0771 ( ${ }^{\star} P=0.049$, Student's $t$-test) and AK4.4 ( ${ }^{\star} P=0.043$, Student's $t$-test). (b) Losartan reduces solid stress in additional models, including $4 \mathrm{~T} 1$ breast tumours $\left({ }^{\star} P=0.036\right.$, Student's $t$ test) and Pan-02 pancreatic tumours $\left({ }^{\star \star} P=0.0092\right.$, Student's $t$-test $)$. Animal numbers $n=5$ (E0771), $n=8-9$ (AK4.4), $n=10-11$ (4T1), $n=4-8$ (Pan-02). Error bars indicate s.e.m.

been assumed that their indirect antiangiogenic properties, through downstream VEGF inhibition, benefit survival additively. Our current findings, in line with our previous report ${ }^{14}$, do not support this antiangiogenic mechanism. Further, we detected no growth delay or survival benefit with losartan monotherapy in our study. This is in contrast to the ability of angiotensin signalling through AT1 to promote tumour growth and metastasis $32,37,38,48,49$, with signalling through AT2 hindering growth ${ }^{50}$. This is perhaps because these growth inhibitory effects are primarily seen at much higher doses of $\mathrm{ARBs}^{36,46,51}$ or with long-term treatment ${ }^{52}$; indeed, when used at low doses, ARBs have been shown by others to not cause a growth delay ${ }^{47}$. It is also possible that this VEGF inhibition is because of direct effects of angiotensin inhibitors on cancer cells, and that our models do not respond similarly because the cancer cells used do not express high levels of AT1 or AT2. These inconsistent data notwithstanding, it should be noted that antiangiogenic therapy with bevacizumab has failed to prolong survival in desmoplastic tumours such as breast and pancreatic cancer $^{3}$, casting doubt on a possible antiangiogenic mechanism for the benefits observed with angiotensin inhibitors.

Here we have found that angiotensin inhibitors actually increase vessel perfusion through vascular decompression, doing so by reducing stromal activity and production of matrix components responsible for compression. Notably, these drugs are the first to target all stromal components (CAFs, hyaluronan and collagen) known to contribute to solid stress ${ }^{5}$. Our findings also suggest that AT2 agonists or inhibitors of downstream signalling through TGF- $\beta 1, \mathrm{CCN} 2$ or ET-1 may similarly reduce solid stress to enhance chemotherapy, although such agents have not been tested in this way. Similarly, angiotensin (1-7), a MAS agonist that can reduce CAF matrix production, may be useful for targeting solid stress ${ }^{53}$. Meanwhile, there are challenges to address for translation. Deleterious effects on blood pressure may contraindicate angiotensin inhibitors for some patients ${ }^{14,54}$. Further, we expect that angiotensin inhibitor distribution into tumours may be the most important factor controlling efficacy: drugs displaying a lack of tissue penetration (such as candesartan) would therefore be poor candidates relative to those with high penetration (such as losartan and telmisartan) ${ }^{55}$. Indeed, the inadequate tumour penetration of candesartan may explain why it only improved chemotherapy outcomes modestly, when comparing high versus low candesartan doses, in a recent pancreatic cancer study ${ }^{54}$. These factors are why losartan was selected over other angiotensin inhibitors for a recently initiated clinical trial in pancreatic cancer (NCT01821729) at 

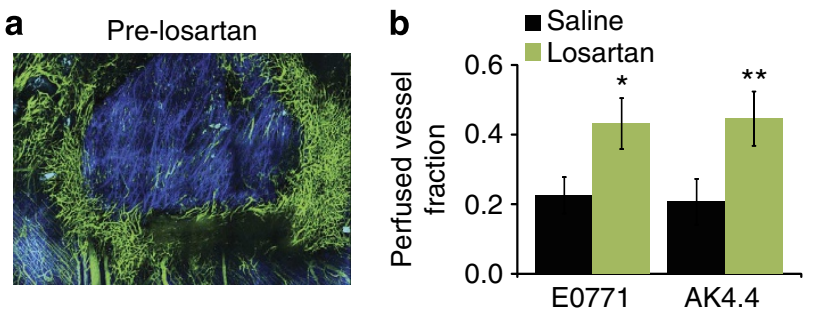

C
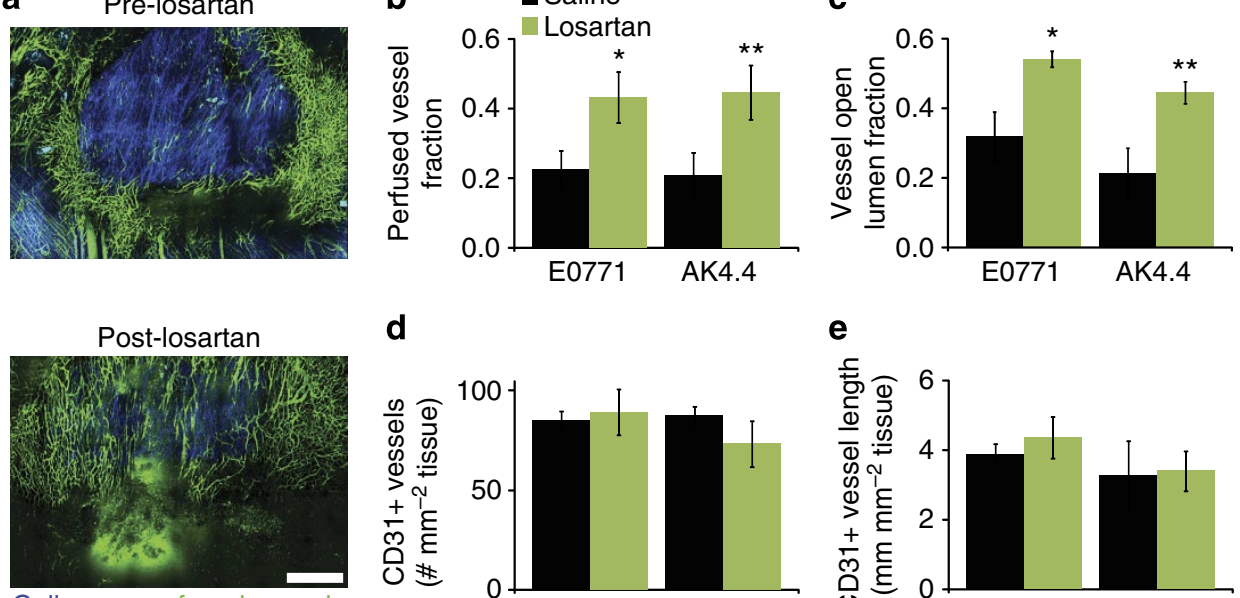

Collagen perfused vessels d

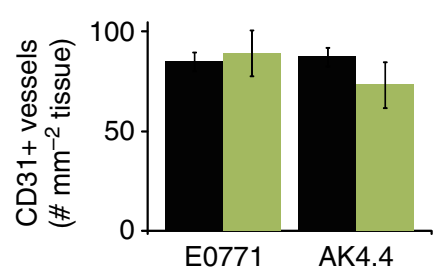

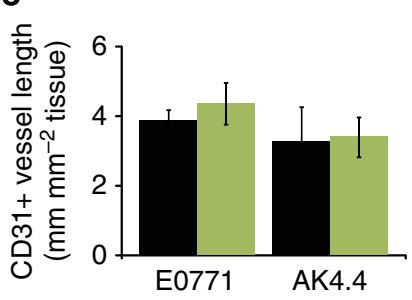

f

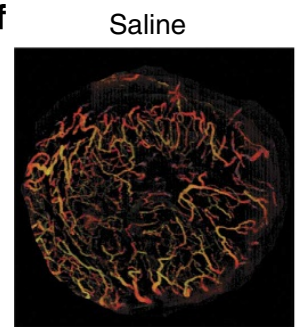

Losartan

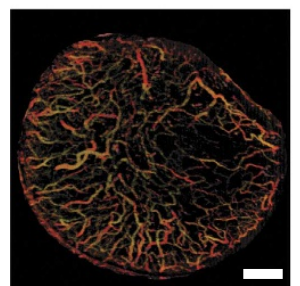

190

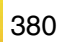

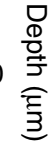

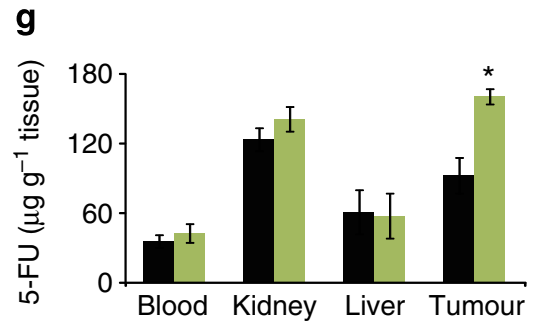

h
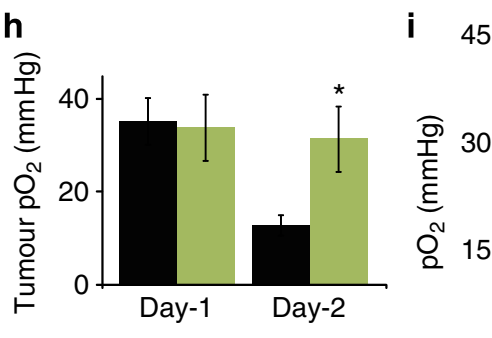

i 45

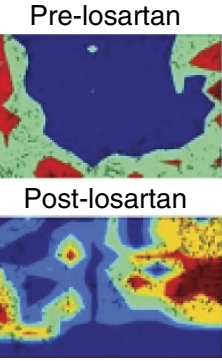

j

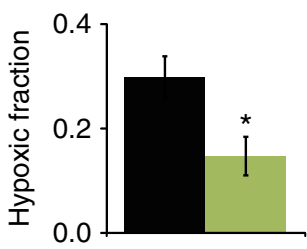

Figure 6 | Losartan decompresses tumour vessels to increase drug and oxygen delivery. (a) Representative images from intravital multiphoton microscopy of perfused tumour vessels (green) and collagen (blue), showing that losartan increases the density of perfused vessels in an E0771 breast tumour. Scale bar, $1 \mathrm{~mm}$. (b) Perfused vessel fractions after angiotensin inhibition using losartan. Losartan increases the fraction of vessels that are perfused in orthotopic E0771 breast ( ${ }^{\star} P=0.038$, Student's $t$-test) and AK4.4 pancreatic ( ${ }^{\star} P=0.039$, Student's $t$-test) tumours. (c) Losartan also increases the fraction of vessels with open lumen in E0771 ( ${ }^{\star} P=0.040$, Student's $t$-test) and AK4.4 ( ${ }^{\star \star} P=0.015$, Student's $t$-test) tumours, indicating decompression as the mechanism ${ }^{9}$. (d) Vessel density and (e) vessel length following angiotensin inhibition using losartan. Losartan does not affect vessel density, as quantified by the vessel number density (d) and the total vessel length (e), indicating no antiangiogenic effect at this $40 \mathrm{mg} \mathrm{kg}{ }^{-1}$ dose. (f) Representative images from intravital optical frequency-domain imaging of perfused vessels with losartan treatment. E0771 tumours in control mice have a low density and poor distribution of perfused vessels in three dimensions, whereas losartan-treated mice showed a more even distribution and higher density of perfused vessels. Scale bar, $1 \mathrm{~mm}$. (g) Small-molecule drug delivery to tumours and various organs after angiotensin inhibition with losartan. Losartan increases the accumulation of the small-molecule chemotherapeutic 5 -FU in AK4.4 pancreatic tumours by $74 \%\left({ }^{\star} P=0.0063\right.$, Student's $t$-test), while not affecting accumulation in the normal organs. (h) Oxygen delivery to tumours measured by phosphorescence quenching microscopy during angiotensin inhibition using losartan, with (i) representative images. Losartan maintains the level of oxygenation (h) in the tissue, versus control tumours that become progressively more hypoxic with time $\left({ }^{\star} P=0.030\right.$, Student's $t$-test $)$ as the tumours grow from 3 to $5 \mathrm{~mm}$ in diameter. Losartan increases oxygenation in some tumours (i) whereas all control tumours decrease in oxygen levels. Losartan also appears to result in a more homogenous distribution of welloxygenated tumour tissue. Scale bar, $100 \mu \mathrm{m}$. (j) Hypoxic fraction in tumours measured by pimonidazole injection and staining following angiotensin inhibition with losartan. Losartan decreases the hypoxic fraction in E0771 tumours ( ${ }^{\star} P=0.019$, Student's $t$-test) because of the increase in oxygen delivery. Animal numbers $n=7-9$ (vessels), $n=4-5$ (vessel lumen), $n=4$ (drug delivery), $n=6$ (oxygen delivery), $n=6-7$ (hypoxia). Error bars indicate s.e.m.

Massachusetts General Hospital. Regardless, the safety and low cost of ARBs and ACE-Is-along with their potentiation of conventional chemotherapy-make a strong case for repurposing angiotensin inhibitors as adjuncts for cancer therapies.

\section{Methods}

Drug preparation. Angiotensin inhibitors (losartan, lisinopril, valsartan and candesartan) were obtained as pills, crushed and dissolved in PBS (for losartan and lisinopril) or PBS with $5 \%$ ethanol (for valsartan and candesartan) over $24 \mathrm{~h}$. The solutions were then sterile filtered for injection. Doxorubicin and 5-FU were obtained as solutions for injection. All drugs were purchased from the pharmacy at Massachusetts General Hospital.

Tumour models. AK4.4 cells ( $\mathrm{rras}^{G 12 D}$ and $p 53^{+/-}$) were kindly provided by Dr Nabeel Bardeesy, and were isolated from mice generating spontaneous pancreatic tumours ${ }^{56}$ (Ptf1-Cre/LSL-Kras $\left.{ }^{G 12 D} / p_{53} 3^{L o x /+}\right)$. Orthotopic pancreatic tumours were generated by implanting a small piece $\left(1 \mathrm{~mm}^{3}\right)$ of viable tumour tissue (from a source tumour in a separate animal) into the pancreas of a 6-8 week-old male FVB (AK4.4 model) or C57BL/6 (Pan-02 model) mouse. Orthotopic breast tumours were similarly generated by implanting a chunk of viable tumour tissue into the mammary fat pad of a 6-8-week-old female severe combined immunodeficient (SCID) mouse (E0771 and 4T1 models) or C57BL/6 mouse (E0771 syngeneic model). For AT1- and AT2-knockout studies, E0771 cells were implanted by injection into the mammary fat pad of 6-8-week-old female C57BL/ 6, agtr1a ${ }^{-1-}$ (ref. 57) or agtr2 $2^{-/-}$(ref. 58) mice. All animal procedures were carried out following the Public Health Service Policy on Humane Care of Laboratory Animals and approved by the Institutional Animal Care and Use Committee of Massachusetts General Hospital.

Vessel perfusion and hypoxia histology. For breast tumours, mice bearing orthotopic E0771 were split into time- and size-matched $\left(\sim 100 \mathrm{~mm}^{3}\right)$ treatment groups. For pancreatic tumours, mice bearing orthotopic AK4.4 were split into treatment groups 6 days post implantation. The mice were then treated with $40 \mathrm{mg} \mathrm{kg}^{-1}$ losartan or an equal volume of PBS intraperitoneally each day for 6 (E0771) or 7 (AK4.4) days. On the day of the last treatment, mice were slowly 
a

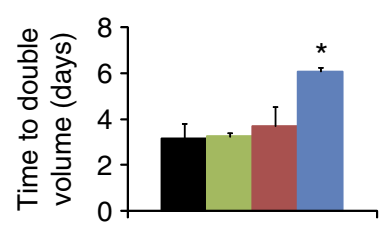

d

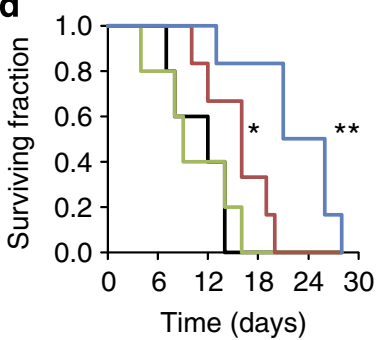

b

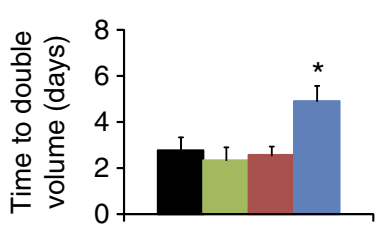

e

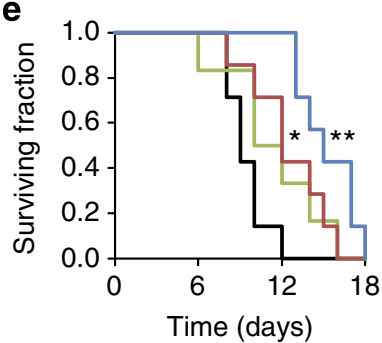

C

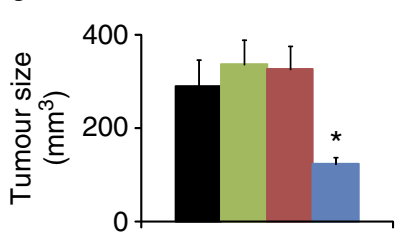

f

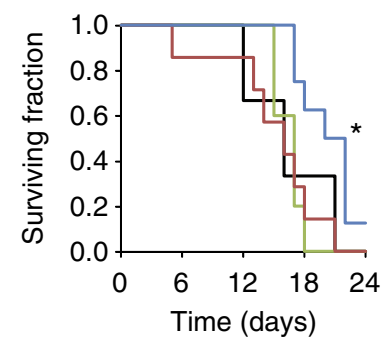

- Saline + saline $\quad$ Saline + chemotherapy

- Losartan + saline Losartan + chemotherapy

Figure 7 | Losartan potentiates chemotherapy. (a) Quantification of tumour growth rates, based on the time to reach double the initial volume, for orthotopic E0771 breast tumours in response to treatment with losartan or saline control $\left(40 \mathrm{mg} \mathrm{kg}^{-1}\right.$ daily from day 0 onwards) in combination with either the small-molecule chemotherapeutic doxorubicin or saline control ( $2 \mathrm{mg} \mathrm{kg}^{-1}$ every 3 days from day 1 onwards). Doxorubicin and losartan monotherapy induce no significant growth delay versus the control treatment in these aggressive tumours. In contrast, their combination greatly limits tumour growth $\left({ }^{\star} P=0.040\right.$, Student's $t$-test). (b) Quantification of tumour growth rates, based on the time to reach double the initial volume, for orthotopic $4 \mathrm{~T} 1$ breast tumours using the same treatments as with E0771. Doxorubicin and losartan monotherapy induce no significant growth delay versus the control treatment. In contrast, their combination greatly limits tumour growth ( ${ }^{\star} P=0.024$, Student's $t$-test). (c) Volumes of orthotopic AK4.4 pancreatic tumours on day 7 in response to treatment with losartan or saline control ( $40 \mathrm{mg} \mathrm{kg}^{-1}$ daily from day 0 to 7 ) in combination with either the small-molecule chemotherapeutic 5-FU or saline control ( $60 \mathrm{mg} \mathrm{kg}^{-1}$ on days 2 and 6 ). 5-FU and losartan monotherapy induce no significant growth delay versus the control treatment, whereas their combination greatly inhibited tumour growth ( ${ }^{\star} P=0.0085$, Student's $t$-test). (d) Animal survival for E0771bearing mice following the initiation of treatment. Doxorubicin monotherapy improves survival versus the control $\left({ }^{\star} P=0.048\right.$, log-rank test), whereas the combination of doxorubicin and losartan enhances this survival increase versus doxorubicin monotherapy $\left({ }^{\star \star} P=0.014\right.$, log-rank test). (e) Animal survival for 4T1-bearing mice following the initiation of treatment. Doxorubicin monotherapy improves survival versus the control ( ${ }^{\star} P=0.045, \log -$ rank test), whereas the combination of doxorubicin and losartan enhances this survival increase versus doxorubicin monotherapy $\left({ }^{\star \star} P=0.050, \log -\mathrm{rank}\right.$ test). (f) Animal survival for AK4.4-bearing mice following the initiation of treatment. The combination of 5-FU and losartan enhances survival versus 5-FU ( ${ }^{\star} P=0.019$, log-rank test) or losartan monotherapy ( ${ }^{\star} P=0.027$, log-rank test). Animal numbers $n=5-6$ (E0771, AK4.4 growth), $n=3-8$ (AK4.4 survival), $n=6-7$ (4T1). Error bars indicate s.e.m. Statistical tests were corrected for multiple comparisons using the Holm-Bonferroni method.

( 2 min) injected with $100 \mu \mathrm{l}$ of $1 \mathrm{mg} \mathrm{ml}^{-1}$ biotinylated lectin (Vector Labs), administered via the retro-orbital sinus 5 min before tumour removal. For hypoxia studies, the mice were also injected with $60 \mathrm{mg} \mathrm{kg}^{-1}$ of pimonidazole at $10 \mathrm{mg} \mathrm{ml}^{-1} 1 \mathrm{~h}$ before tumour removal. The tumours were then excised, fixed in $4 \%$ formaldehyde in PBS ( $30 \mathrm{~min} \mathrm{~mm}^{-1}$ diameter of tissue), incubated in $30 \%$ sucrose in PBS overnight at $4{ }^{\circ} \mathrm{C}$ and frozen in optimal cutting temperature compound (Tissue-Tek). Transverse tumour sections, $40 \mu \mathrm{m}$ thick, were immunostained with antibodies to endothelial marker CD31 (MEC13.3 antibody, Biosciences (BD), 1:100 dilution) and counterstained with 4',6-diamidino-2-phenylindole (Vector Labs). Collagen I and hyaluronan were, respectively, detected using the LF-68 antibody (1:50 dilution) provided by Dr Larry Fisher (NIDCR) and a biotinylated hyaluronan proteoglycan fragment (385911, Calbiochem), as previously described ${ }^{40}$. Staining for $\alpha$ SMA (C6198 antibody, Sigma, 1:100 dilution), active TGF- $\beta 1$ (G122A antibody, Promega, 1:15 dilution), CCN2 (TP-243 antibody, Torrey Pines, 1:100 dilution), AT1 (ab18801 antibody, Abcam, 1:100 dilution) and AT2 (AAR-012 antibody, Alomone, 1:200 dilution) were carried out in $10 \mu \mathrm{m}$ sections. For the detection of collagen I in AK4.4, KPC and human pancreatic ductal adenocarcinoma, the paraffin-embedded sections were treated with a pH-9.0 antigen retrieval solution and counterstained with haematoxylin.

Histological image analysis. Eight random fields (four interior and four periphery) at $\times 20$ magnification were taken from each slide using a confocal microscope (Olympus). For vascular analysis, vessels were skeletonized and segmented using a custom, semi-automated tracing program developed in MATLAB (The MathWorks), allowing the removal of structures under 30 pixels and regions of autofluorescence. For perfusion fraction, the number of vessels counted by this program with colocalization of lectin and CD31 staining was divided by the number of vessels counted with CD31 staining. For vessel metrics, including diameter and density, the program determined the average size of all counted vessels and their length, as well as the count per area. For open versus closed lumen quantification, vessels were analysed by eye and a vessel was counted as open if it had a clearly visible lumen throughout its length. Images of collagen I, hyaluronan, pimonidazole (hypoxia) $\alpha \mathrm{SMA}$, active-TGF- $\beta 1$ and CCN2-stained sections were analysed based on the area fraction of positive staining. Identical analysis settings and thresholds were used for all tumours.

CAF studies. We generated $\alpha S M A^{P}$ - $d s$ Red/Tie $2^{P}$-GFP/FVB double-transgenic mice by crossing Tie $2^{P}$-GFP/FVB mice ${ }^{59}$ with $\alpha S M A^{P}$-dsRed mice ${ }^{19}$. Once established, this line was backcrossed to FVB mice for at least 10 generations. CAFs were isolated from AK4.4 tumours implanted in 6-8-week-old male mice of this line. The mice were treated with $40 \mathrm{mg} \mathrm{kg}^{-1}$ losartan or PBS for 7 days, and then the tumours were collected and $\alpha \mathrm{SMA}+$ cells were isolated based on dsRed fluorescence using fluorescence-activated cell sorting (BD Biosciences). These cells were analysed with quantitative reverse transcriptase-PCR for messenger RNA of Colla1 (F 5'-GCTCCTCTTAGGGGCCACT-3', R 5'-CCACGTCTCACCATTGG GG-3'), Hasl (F, 5'-GGCGAGCACTCACGATCATC-3', R 5'-AGGAGTCCATA GCGATCTGAAG-3'), Has2 (F 5'-TGTGAGAGGTTTCTATGTGTCCT-3', R 5'ACC GTA CAG TCC AAA TGA GAA GT-3'), Has 3 (F $5^{\prime}$-CCTGGAGCACCGT CGAATG-3', R $5^{\prime}$-CCTTGAGGTTTGGAAAGGCAA-3'), Tgfb1 (F $5^{\prime}$-GCAGTG GCTGAACCAAGGA-3', R 5'-AGCAGTGAGCGCTGAATCG-3'), Ctgf (F 5'-CAA AGCAGCTGCAAATACCA- $3^{\prime}$, R $5^{\prime}$-GTCTGGGCCAAATGTGTCTT- $3^{\prime}$ ) and Edn1 (F 5'-GCACCGGAGCTGAGAATGG-3', R 5'-GTGGCAGAAGTAGACAC ACTC- $\left.3^{\prime}\right)$. We also analysed these cells, as well as AK4.4 and E0771 cells, for Agtrla (F 5'-AACAGCTTGGTGGTGATCGTC-3', R 5'-CATAGCGGTATAGAC AGCCCA-3'), Agtr1b (F 5'-TGGCTTGGCTAGTTTGCCG-3', R 5' -ACCCAGTCC AATGGGGAGT-3') and Agtr2 (F 5'-AACTGGCACCAATGAGTCCG-3', R 5'-CCAAAAGGAGTAAGTCAGCCAAG-3'). Expression levels were normalized to the control (GAPDH) and losartan-treated levels were normalized to the PBS controls.

Solid stress. Solid stress was measured using the tumour opening technique ${ }^{5}$. When the tumours reached a size of $\sim 1 \mathrm{~cm}$ in diameter, the mice were anaesthetized. Subsequently, each tumour was excised, washed with Hank's balanced salt solution and its three dimensions were measured. Each tumour was 
cut along its longest axis, to a depth of $80 \%$ of its shortest dimension, using a scalpel. The tumours were allowed to relax for $10 \mathrm{~min}$ in Hank's balanced salt solution to diminish any transient, poro-elastic responses. Afterwards, the opening resulting from the cut was measured at the middle of the cut at the surface of the tumour. Solid stress is proportional to the size of the opening relative to the size of the dimension perpendicular to the cut.

Drug delivery. Mice bearing orthotopic AK4.4 were split into treatment groups 6 days after implantation. The mice were then treated with $40 \mathrm{mg} \mathrm{kg}^{-1}$ losartan or an equal volume of PBS intraperitoneally each day for 7 days. On the day of the last treatment, mice were injected with $100 \mathrm{mg} \mathrm{kg}^{-1}$ 5-FU, administered retro-orbitally $30 \mathrm{~min}$ before tumour and organ removal. The tissue was dabbed of excess blood and then snap-frozen in liquid nitrogen for analysis. 5-FU was isolated from the tissues and measured using liquid-liquid extraction followed by reverse-phase HPLC with tandem mass-spectrometry.

In vivo imaging. For imaging studies, E0771 tumours were implanted in mice bearing mammary fat pad chambers ${ }^{33}$ and allowed to grow to $\sim 3 \mathrm{~mm}$ in diameter. Multiphoton imaging was carried out on a custom-built multiphoton laserscanning microscope using a confocal laser-scanning microscope body (Olympus 300, Optical Analysis) and a broadband femtosecond laser source (High Performance MaiTai, Spectra-Physics ${ }^{60}$. Images were taken at $\sim 60 \mathrm{~mW}$ at sample surface. Mosaic images were taken in raster pattern using a motorized stage (H101, Prior Scientific, Inc.) and customized automation software (LabView, National Instruments). Imaging studies were performed with a $\times 20$ magnification, $0.95 \mathrm{NA}$ water immersion objective (Olympus XLUMPlanFl, 1-UB965, Optical Analysis). Optical frequency-domain imaging was carried out after 7 days of treatments using a custom-built microscope ${ }^{61}$.

Tissue oxygenation. We measured $\mathrm{pO} 2$ in the tumours using phosphorescence quenching microscopy ${ }^{35}$, which was adapted to multiphoton microscopy. An oxygen-sensitive porphyrin, Oxyphor R2 (Oxygen Enterprises) was injected retroorbitally $12 \mathrm{~h}$ before imaging, and was reinjected immediately before imaging along with $2 \mathrm{MDa}$ fluorescein isothiocyanate-dextran (Sigma-Aldrich) for functional vascular tracing. A mosaic image of the tumour was collected, and oxygen was then measured in an evenly spaced $12 \times 12$ grid at four depths $(60,120,180$ and $240 \mu \mathrm{m}$ ) in the tumour. At each point in the grid, the phosphorescence lifetime of the probe was measured after each of several repeated brief intense pulses of $1,020 \mathrm{~nm}$ laser light, and these lifetime measurements were combined. A twocomponent model was used to calculate the oxygen tension from each lifetime measurement, accounting for binding and quenching of the probe by both oxygen and proteins. The grid was then overlaid on the mosaic to make an oxygen map.

Effective permeability. Effective permeability (transvascular flux) studies were carried out using a mixture of nanoparticles with diameters of $12 \mathrm{~nm}(476 \mathrm{~nm}$ emission), $60 \mathrm{~nm}\left(540 \mathrm{~nm}\right.$ emission) and $125 \mathrm{~nm}(625 \mathrm{~nm} \text { emission })^{33}$. Concentrations were adjusted with in vitro calibration to result in roughly equal photoluminescence intensity for all three nanoparticle samples under $800 \mathrm{~nm}$ multiphoton excitation. Following retro-orbital injection of $200 \mu \mathrm{l}$ with these concentrations, multiphoton imaging was carried out as described above at depths from 0 to $201 \mu \mathrm{m}$, with $2.76 \mu \mathrm{m}$ steps and $2.76 \times 2.76 \mu \mathrm{m}$ pixels. Images were taken every $3 \mathrm{~min}$ at each region of interest for a duration of $1 \mathrm{~h}$. Images were analysed using custom analysis software developed in Matlab (The MathWorks) ${ }^{33,62}$. The analysis approach involved three-dimensional (3D) vessel tracing to create vessel metrics and a 3D map of voxel intensity versus distance to the nearest vessel over time. Images were also corrected for sample movement over time with 3D image registration. The effective permeability (normalized transvascular flux) was

calculated using $\frac{J \mathrm{t}}{S_{\mathrm{v}}\left(C_{\mathrm{v}}-C\right)}=P_{\mathrm{ef} f}=\lim _{t \rightarrow 0} \frac{\partial}{\partial t} \frac{\int_{r=R}^{\infty} C(r) r \partial r}{\left(C_{\mathrm{v}}-C\right) R}$, where $J_{\mathrm{t}}$ is the transvascular flux, $S_{\mathrm{v}}$ is the vessel surface area, $C_{\mathrm{v}}$ is the concentration of the probe in the vessel, $C$ is the concentration of the probe immediately extravascular, $P_{\text {eff }}$ is the effective permeability, $t$ is the time after the initial image, $r$ is the distance from the vessel central axis and $R$ is the vessel radius at that point along the vessel. Fluorescence intensities were used as these concentrations. The calculation was made as an average over the entire imaged volume for each tumour.

Individual tumour vessel blood flow. Red blood cell (RBC) velocity was measured using intravital microscopy with the residence time line scanning technique in mice bearing orthotopic E0771 or 4T1 mammary tumours ${ }^{63}$. RBCs from a donor mouse were labelled ex vivo with $1,1^{\prime}$-dioctadecyl- $3,3,3^{\prime}, 3^{\prime}$ -

tetramethylindodicarbocyanine perchlorate. These labelled RBCs were administered to mice bearing orthotopic E0771 or 4T1 mammary tumours via retro-orbital injection at a ratio of 3-5 labelled RBCs per 100 RBCs. Multiphoton imaging was carried out, applying laser scanning along a single line that intersects each vessel of interest. Repeated scanning along this line generated fluorescence intensity data along the line over time $(x-t)$, in which 'images' of the fluorescent RBCs are compressed or elongated depending on the residence time of the cells in the scan. Velocities were extracted using $v_{\mathrm{rbc}}=\frac{L_{\mathrm{rbc}} \cdot f}{n_{\mathrm{casc}} \cdot \sin (\alpha)}$, where $v_{\mathrm{rbc}}$ is the RBC velocity, $L_{\mathrm{rbc}}$ is the RBC length $(7 \mu \mathrm{m}), f$ is the line scan frequency (scans $\mathrm{s}^{-1}$ ), $n_{\text {scans }}$ is the number of consecutive scan lines that detected the RBC and $\alpha$ is the angle between vessel and line scan.

Breast tumour growth and survival studies. Mice bearing orthotopic E0771 or 4T1 breast tumours were split into treatment groups, time-matched for time after implantation and size-matched for tumour volume at this time $\left(105 \mathrm{~mm}^{3}\right.$ in E0771 and $141 \mathrm{~mm}^{3}$ in 4T1). The mice were then treated with $40 \mathrm{mg} \mathrm{kg}^{-1}$ losartan or an equal volume of PBS intraperitoneally on day 0 and each subsequent day. The mice were then treated with either $2 \mathrm{mg} \mathrm{kg}^{-1}$ (SCID) or $5 \mathrm{mg} \mathrm{kg}^{-1}$ (C57BL/6) doxorubicin, or an equal volume of saline, by intraperitonteal injection every 3 days beginning on day 1 (after two losartan or PBS treatments). Doxorubicin or control treatments were carried out $4 \mathrm{~h}$ after losartan or control treatments. The primary tumours were then measured every 1-2 days, beginning on day 0 , using calipers. Tumour growth was quantified using the time for each to reach double (SCID) or triple (C57BL/6) its initial volume. Animal survival was quantified based on the time of death after initiation of treatment or time to reach excessive tumour burden $\left(1000 \mathrm{~mm}^{3}\right)$. For metastasis studies, mouse lungs were collected at the killing and were fixed in Bouin's solution. Macrometastases were counted using a stereomicroscope.

Pancreatic tumour growth and survival studies. Mice bearing orthotopic AK4.4 pancreatic tumours were split into treatment groups, size-matched for tumour volume $\left(22 \mathrm{~mm}^{3}\right)$ by surgical exposure, 6 days after implantation. The mice were treated with $40 \mathrm{mg} \mathrm{kg}^{-1}$ losartan or an equal volume of PBS intraperitoneally on day 7 after implantation and each subsequent day. The mice were then treated with either $60 \mathrm{mg} \mathrm{kg}^{-1} 5$-FU or an equal volume of saline by intravenous injection on days 9 and 13 after implantation. Tumours were extracted on day 14 for measurement using calipers. Tumour growth was quantified using the size at day 14. For survival studies, mice were treated daily with losartan or PBS starting on day 3 after implantation until day 13, and then with 5-FU or saline on days 5, 9 and 13 .

Mean arterial pressure. Mice bearing orthotopic AK4.4 pancreatic tumours were used for blood pressure measurements. Mean arterial pressure was measured by cannulation of the left carotid artery after a longitudinal skin incision above the trachea $^{14}$. After removal of the submandibular gland, the paratracheal muscles were split and the left carotid artery was isolated. The cranial end of the artery was ligated with a 6-0 silk suture and another suture was tied loosely around the central part of the artery. A metal clamp was then positioned caudally to stop blood flow during the cannulation. A polyethylene catheter (PE-10, Becton-Dickinson) filled with heparinized saline was then inserted through a hole cut proximally to the cranial ligature, and the other suture was tied tightly around the tubing and artery The clamp was then removed and the end of the tubing was connected to a pressure transducer for the measurement of blood pressure.

Mathematical analysis and modelling. The analysis was carried out on mosaic images of whole tumours taken with multiphoton microscopy after injection of $2 \mathrm{MDa}$ fluorescein isothiocyanate-dextran as a perfused vessel tracer. Details of the models and corresponding equations are described in Baish et $a l^{34}$.

Statistical analysis. The data are presented as means with s.e. Groups were compared using a Student's $t$-test, paired or unpaired, except for animal survival studies where a log-rank test was used. In pairwise comparisons within studies where multiple comparisons were made, $P$-values were adjusted using Holm's method.

\section{References}

1. Chauhan, V. P., Stylianopoulos, T., Boucher, Y. \& Jain, R. K. Delivery of molecular and nanoscale medicine to tumors: transport barriers and strategies. Ann. Rev. Chem. Biomol. Eng. 2, 281-298 (2011).

2. Tsai, A. G., Johnson, P. C. \& Intaglietta, M. Oxygen gradients in the microcirculation. Physiol. Rev. 83, 933-963 (2003).

3. Jain, R. K. Normalizing tumor microenvironment to treat cancer: bench to bedside to biomarkers. J. Clin. Oncol. 31, 2205-2218 (2013).

4. Helmlinger, G., Netti, P. A., Lichtenbeld, H. C., Melder, R. J. \& Jain, R. K. Solid stress inhibits the growth of multicellular tumor spheroids. Nat. Biotechnol. 15, 778-783 (1997).

5. Stylianopoulos, T. et al. Causes, consequences, and remedies for growthinduced solid stress in murine and human tumors. Proc. Natl Acad. Sci. USA 109, 15101-15108 (2012)

6. Stylianopoulos, T. et al. Coevolution of solid stress and interstitial fluid pressure in tumors during progression: Implications for vascular collapse. Cancer Res. 73, 3833-3841 (2013).

7. Janmey, P. A. \& McCulloch, C. A. Cell mechanics: integrating cell responses to mechanical stimuli. Annu. Rev. Biomed. Eng. 9, 1-34 (2007).

8. Griffon-Etienne, G., Boucher, Y., Brekken, C., Suit, H. D. \& Jain, R. K. Taxaneinduced apoptosis decompresses blood vessels and lowers interstitial fluid 
pressure in solid tumors: clinical implications. Cancer Res. 59, 3776-3782 (1999).

9. Padera, T. P. et al. Pathology: cancer cells compress intratumour vessels. Nature 427, 695 (2004).

10. Park, M. S. et al. Perfusion CT: noninvasive surrogate marker for stratification of pancreatic cancer response to concurrent chemo- and radiation therapy. Radiology 250, 110-117 (2009).

11. Sorensen, A. G. et al. Increased survival of glioblastoma patients who respond to antiangiogenic therapy with elevated blood perfusion. Cancer Res. 72, 402-407 (2012).

12. Provenzano, Paolo P. et al. Enzymatic targeting of the stroma ablates physical barriers to treatment of pancreatic ductal adenocarcinoma. Cancer Cell 21, 418-429 (2012).

13. Alexandrakis, G. et al. Two-photon fluorescence correlation microscopy reveals the two-phase nature of transport in tumors. Nat. Med. 10, 203-207 (2004).

14. Diop-Frimpong, B., Chauhan, V. P., Krane, S., Boucher, Y. \& Jain, R. K. Losartan inhibits collagen I synthesis and improves the distribution and efficacy of nanotherapeutics in tumors. Proc. Natl Acad. Sci. USA 108, 2909-2914 (2011).

15. Liu, J. et al. TGF- $\beta$ blockade improves the distribution and efficacy of therapeutics in breast carcinoma by normalizing the tumor stroma. Proc. Natl Acad. Sci. USA 109, 16618-16623 (2012).

16. Naito, T. et al. Angiotensin II induces thrombospondin-1 production in human mesangial cells via p38 MAPK and JNK: a mechanism for activation of latent TGF- $\beta 1$. Am. J. Physiol. 286, F278-F287 (2004).

17. Habashi, J. P. et al. Losartan, an AT1 antagonist, prevents aortic aneurysm in a mouse model of Marfan syndrome. Science 312, 117-121 (2006).

18. Sweetwyne, M. T. \& Murphy-Ullrich, J. E. Thrombospondin1 in tissue repair and fibrosis: TGF-beta-dependent and independent mechanisms. Matrix Biol. 31, 178-186 (2012).

19. Liao, S. et al. Impaired lymphatic contraction associated with immunosuppression. Proc. Natl Acad. Sci. USA 108, 18784-18789 (2011).

20. Mori, T. et al. Role and interaction of connective tissue growth factor with transforming growth factor-beta in persistent fibrosis: A mouse fibrosis model. J. Cell Physiol. 181, 153-159 (1999).

21. Duncan, M. R. et al. Connective tissue growth factor mediates transforming growth factor beta-induced collagen synthesis: downregulation by cAMP. FASEB J. 13, 1774-1786 (1999).

22. Rodriguez-Vita, J. et al. Angiotensin II activates the Smad pathway in vascular smooth muscle cells by a transforming growth factor-beta-independent mechanism. Circulation 111, 2509-2517 (2005).

23. Yang, F., Chung, A. C., Huang, X. R. \& Lan, H. Y. Angiotensin II induces connective tissue growth factor and collagen I expression via transforming growth factor-beta-dependent and -independent Smad pathways: the role of Smad3. Hypertension 54, 877-884 (2009).

24. Hama, K. et al. Angiotensin II stimulates DNA synthesis of rat pancreatic stellate cells by activating ERK through EGF receptor transactivation. Biochem. Biophys. Res. Commun. 315, 905-911 (2004).

25. Liu, W. B., Wang, X. P., Wu, K. \& Zhang, R. L. Effects of angiotensin II receptor antagonist, Losartan on the apoptosis, proliferation and migration of the human pancreatic stellate cells. World J. Gastroenterol. 11, 6489-6494 (2005).

26. Leask, A. Targeting the TGF beta, endothelin-1 and CCN2 axis to combat fibrosis in scleroderma. Cell Signal. 20, 1409-1414 (2008).

27. Shi-Wen, X. et al. Constitutive ALK5-independent c-Jun N-terminal kinase activation contributes to endothelin- 1 overexpression in pulmonary fibrosis: evidence of an autocrine endothelin loop operating through the endothelin A and B receptors. Mol. Cell Biol. 26, 5518-5527 (2006).

28. Cheng, T. H. et al. Involvement of reactive oxygen species in angiotensin IIinduced endothelin-1 gene expression in rat cardiac fibroblasts. J. Am. Coll. Cardiol. 42, 1845-1854 (2003).

29. Habashi, J. P. H. J. P. et al. Angiotensin II type 2 receptor signaling attenuates aortic aneurysm in mice through ERK antagonism. Science 332, 361-365 (2011).

30. Ulmasov, B., Xu, Z., Tetri, L. H., Inagami, T. \& Neuschwander-Tetri, B. A. Protective role of angiotensin II type 2 receptor signaling in a mouse model of pancreatic fibrosis. Am. J. Physiol. Gastroint. Liver Physiol. 296, G284-G294 (2009).

31. Nabeshima, Y. et al. Anti-fibrogenic function of angiotensin II type 2 receptor in CCl4-induced liver fibrosis. Biochem. Biophys. Res. Commun. 346, 658-664 (2006).

32. George, A. J., Thomas, W. G. \& Hannan, R. D. The renin-angiotensin system and cancer: old dog, new tricks. Nat. Rev. Cancer 10, 745-759 (2010).

33. Chauhan, V. P. et al. Normalization of tumour blood vessels improves the delivery of nanomedicines in a size-dependent manner. Nat. Nanotechnol. 7, 383-388 (2012).

34. Baish, J. W. et al. Scaling rules for diffusive drug delivery in tumor and normal tissues. Proc. Natl Acad. Sci. USA 108, 1799-1803 (2011).

35. Helmlinger, G., Yuan, F., Dellian, M. \& Jain, R. K. Interstitial pH and pO2 gradients in solid tumors in vivo: high-resolution measurements reveal a lack of correlation. Nat. Med. 3, 177-182 (1997).
36. Arnold, S. A. et al. Losartan slows pancreatic tumor progression and extends survival of SPARC-null mice by abrogating aberrant TGF $\beta$ activation. PLoS One 7, e31384 (2012).

37. Rodrigues-Ferreira, S. et al. Angiotensin II facilitates breast cancer cell migration and metastasis. PLoS One 7, e35667 (2012).

38. Amano, H. et al. Angiotensin II type 1A receptor signaling facilitates tumor metastasis formation through P-selectin-mediated interaction of tumor cells with platelets and endothelial cells. Am. J. Pathol. 182, 553-564 (2013).

39. DeNardo, D. G. et al. Leukocyte complexity predicts breast cancer survival and functionally regulates response to chemotherapy. Cancer Discov. 1, 54-67 (2011).

40. Pluen, A. et al. Role of tumor-host interactions in interstitial diffusion of macromolecules: cranial vs. subcutaneous tumors. Proc. Natl Acad. Sci. USA 98, 4628-4633 (2001).

41. Keizman, D. et al. Angiotensin system inhibitors and outcome of sunitinib treatment in patients with metastatic renal cell carcinoma: A retrospective examination. Eur. J. Cancer 47, 1955-1961 (2011).

42. Nakai, Y. et al. Inhibition of renin-angiotensin system affects prognosis of advanced pancreatic cancer receiving gemcitabine. Br. J. Cancer 103, 1644-1648 (2010).

43. Wilop, S. et al. Impact of angiotensin I converting enzyme inhibitors and angiotensin II type 1 receptor blockers on survival in patients with advanced non-small-cell lung cancer undergoing first-line platinum-based chemotherapy. J. Cancer Res. Clin. Oncol. 135, 1429-1435 (2009).

44. Chae, Y. K. et al. Reduced risk of breast cancer recurrence in patients using ACE inhibitors, ARBs, and/or statins. Cancer Invest. 29, 585-593 (2011).

45. Fujita, M. et al. Angiotensin type 1a receptor signaling-dependent induction of vascular endothelial growth factor in stroma is relevant to tumor-associated angiogenesis and tumor growth. Carcinogenesis 26, 271-279 (2005).

46. Suganuma, T. et al. Functional expression of the angiotensin II type 1 receptor in human ovarian carcinoma cells and its blockade therapy resulting in suppression of tumor invasion, angiogenesis, and peritoneal dissemination. Clin. Cancer Res. 11, 2686-2694 (2005).

47. Yoshiji, H. et al. The angiotensin-I-converting enzyme inhibitor perindopril suppresses tumor growth and angiogenesis: Possible role of the vascular endothelial growth factor. Clin. Cancer Res. 7, 1073-1078 (2001).

48. Greco, S. et al. Angiotensin II activates extracellular signal regulated kinases via protein kinase $\mathrm{C}$ and epidermal growth factor receptor in breast cancer cells. J. Cell Physiol. 196, 370-377 (2003).

49. Egami, K. et al. Role of host angiotensin II type 1 receptor in tumor angiogenesis and growth. J. Clin. Invest. 112, 67-75 (2003).

50. Doi, C. et al. Angiotensin II type 2 receptor signaling significantly attenuates growth of murine pancreatic carcinoma grafts in syngeneic mice. BMC Cancer 10, 67 (2010).

51. Rhodes, D. R. et al. AGTR1 overexpression defines a subset of breast cancer and confers sensitivity to losartan, an AGTR1 antagonist. Proc. Natl Acad. Sci. USA 106, 10284-10289 (2009).

52. Noguchi, R. et al. Synergistic inhibitory effect of gemcitabine and angiotensin type-1 receptor blocker, losartan, on murine pancreatic tumor growth via antiangiogenic activities. Oncol. Rep. 22, 355-360 (2009).

53. Cook, K. L., Metheny-Barlow, L. J., Tallant, E. A. \& Gallagher, P. E. Angiotensin-(1-7) reduces fibrosis in orthotopic breast tumors. Cancer Res. 70, 8319-8328 (2010).

54. Nakai, Y. et al. A multicenter phase II trial of gemcitabine and candesartan combination therapy in patients with advanced pancreatic cancer: GECA2. Invest. N. Drugs. doi:10.1007/s10637-013-9972-5, (2013).

55. Michel, M. C., Foster, C., Brunner, H. R. \& Liu, L. A systematic comparison of the properties of clinically used angiotensin II type 1 receptor antagonists. Pharmacol. Rev. 65, 809-848 (2013).

56. Bardeesy, N. et al. Both p16(Ink4a) and the p19(Arf)-p53 pathway constrain progression of pancreatic adenocarcinoma in the mouse. Proc. Natl Acad. Sci. USA 103, 5947-5952 (2006).

57. Sugaya, T. et al. Angiotensin II type 1a receptor-deficient mice with hypotension and hyperreninemia. J. Biol. Chem. 270, 18719-18722 (1995).

58. Ichiki, T. et al. Effects on blood pressure and exploratory behaviour of mice lacking angiotensin II type-2 receptor. Nature 377, 748-750 (1995).

59. Motoike, T. et al. Universal GFP reporter for the study of vascular development. Genesis 28, 75-81 (2000).

60. Brown, E. B. et al. In vivo measurement of gene expression, angiogenesis and physiological function in tumors using multiphoton laser scanning microscopy. Nat. Med. 7, 864-868 (2001).

61. Vakoc, B. J. et al. Three-dimensional microscopy of the tumor microenvironment in vivo using optical frequency domain imaging. Nat. Med. 15, 1219-1223 (2009).

62. Chauhan, V. P. et al. Fluorescent nanorods and nanospheres for real-time in vivo probing of nanoparticle shape-dependent tumor penetration. Angew. Chem. Int. Ed. 50, 11417-11420 (2011).

63. Kamoun, W. S. et al. Simultaneous measurement of RBC velocity, flux, hematocrit and shear rate in vascular networks. Nat. Methods 7, 655-660 (2010). 


\section{Acknowledgements}

We thank Julia Kahn, Sylvie Roberge, Christina Koppel, Carolyn Smith and Eve Smith for technical assistance. We also thank Drs Dan G. Duda, Jeffrey Clark and Pieter Timmermans for helpful discussions, and Dr Nabeel Bardeesy for providing us with AK4.4 cells. This research was primarily supported by the U.S. National Cancer Institute Grants P01CA080124, R01-CA126642, R01-CA085140, R01-CA115767 and R01-CA098706, and by the U.S. Department of Defense Breast Cancer Research Program Innovator Award W81XWH10-1-0016. Additional support was provided by a Dana-Farber Harvard Cancer Center/MIT Koch Institute Bridge Project grant from the Lustgarten Foundation. V.P.C. is supported by a Ruth L. Kirschstein NRSA Post-doctoral Fellowship from the NIH, T32-CA073479.

\section{Author contributions}

V.P.C., J.D.M. and R.K.J. designed the study. V.P.C., J.D.M., H.L., D.A.L., S.R.J., S.V.K., T.S., A.S.M., X.H., P.A., Z.P., P.H. and Y.B. performed research. V.P.C., J.D.M., T.S., Y.B. and R.K.J. analysed data. Z.P., P.H. and M.G.B. developed animal models and reagents. V.P.C., J.D.M., Y.B. and R.K.J. wrote the manuscript.

\section{Additional information}

Supplementary Information accompanies this paper at http://www.nature.com/ naturecommunications
Competing financial interests: R.K.J. received consultant fees from Enlight, Noxxon, Zyngenia and WebMD. R.K.J owns equity in Enlight, SynDevRx and XTuit, and serves on the Board of Directors of XTuit and Boards of Trustees of H\&Q Healthcare Investors and H\&Q Life Sciences Investors. V.P.C. received consultant fees and owns equity in XTuit. Y.B. received consultant fees from XTuit. The Massachusetts General Hospital has applied for a patent based on this work, titled 'Novel compositions and uses of anti-hypertension agents for cancer therapy' (PCT/US2011/061510), with V.P.C., Y.B. and R.K.J. as co-authors. The remaining authors declare no competing financial interests.

Reprints and permission information is available online at http://npg.nature.com/ reprintsandpermissions/

How to cite this article: Chauhan, V.P. et al. Angiotensin inhibition enhances drug delivery and potentiates chemotherapy by decompressing tumour blood vessels. Nat. Commun. 4:2516 doi: 10.1038/ncomms3516 (2013).

(c) (1) $\Theta$ This work is licensed under a Creative Commons AttributionBY NG ND NonCommercial-NoDerivs 3.0 Unported License. To view a copy of this license, visit http://creativecommons.org/licenses/by-nc-nd/3.0/ 